\title{
IS NARCISSISM ASSOCIATED WITH WORKAHOLISM? THE MODERATING ROLE OF WORKLOAD
}

\author{
Alessandra Falco, Annamaria Di Sipio, Damiano Girardi, Vincenzo Calvo, \\ \& Cristina Marogna \\ FISPPA Section of Applied Psychology, University of Padova (Italy)
}

\begin{abstract}
Workaholism, or the tendency to work excessively hard on a compulsive basis, is a form of heavy work investment that is mostly associated with negative consequences for both the individual and the organization. According to the biopsychosocial model, workaholism has a multifactorial genesis. From this standpoint, it may stem from a complex interaction between individual (e.g., personality traits) and situational (e.g., organizational) factors. Among individual factors, narcissism may play a central role. Narcissism can be seen as a set of personality traits that imply a grandiose but fragile sense of self and entitlement, preoccupation with success as well as demands for admiration. Individuals with high levels of narcissism may dedicate considerable time and effort to their work, given that they pursue power, status, social recognition, and rewards in the workplace. For these reasons, we expect narcissism to be positively associated with workaholism. With respect to organizational factors, workload signals what the norms are in an organization (i.e., to work exceedingly hard). Hence, high workload may encourage workaholism in individuals with strong narcissistic components, who may feel compelled to work hard to achieve social recognition and rewards, as well as to attain ego enhancement, by meeting these extremely demanding external standards. In this study, we hypothesized that narcissism is positively associated with workaholism (Hypothesis 1), and that workload may exacerbate this association, which is expected to be stronger when workload is high (Hypothesis 2). The study was carried out on a sample of 217 workers who completed a self-report questionnaire aimed at determining narcissism, workload, and workaholism, in terms of working excessively (WE) and working compulsively (WC). Data were analyzed using moderated multiple regression. Results showed that narcissism was not associated with either workaholism (overall score) or its components, namely WE and WC (Hypothesis 1 not supported). Furthermore, workload moderated the association between narcissism and workaholism (overall score as well as WE/WC), which was significant only when workload was high (Hypothesis 2 supported). Overall, in line with the biopsychosocial model's prediction, this study suggests that workaholism may result from an interaction between individual predispositions, such as narcissism, and organizational factors that encourage employees to work hard (e.g., high workload). Finally, from a practical standpoint, this study recommends that organizations should target narcissism, especially in managers. Indeed, narcissistic leaders, who necessarily face high job demands in their work, are particularly at risk of workaholism and may also encourage workaholism in their subordinates.
\end{abstract}

Keywords: Workaholism, narcissism, workload, moderation.

\section{Introduction}

Narcissism has received growing attention in organizational contexts, especially with respect to issues such as leadership, perceptions of organizational justice, and job performance (Campbell, Hoffman, Campbell, \& Marchisio, 2011). Quite surprisingly, although with some exceptions (Andreassen, Ursin, Eriksen, \& Pallesen, 2012; Clark, Lelchook, \& Taylor, 2010), little research has investigated the relationship between narcissism and heavy work investment (HWI), a concept that implies an elevated investment of both time and effort in one's work (Snir \& Harpaz, 2012). To contribute to fill this gap in the literature, in this study we focused on the association between narcissism and workaholism, a "bad" form of HWI (Schaufeli, 2016) that is mostly associated with negative consequences for both the individual and the organization, such as reduced job and life satisfaction, physical and psychological symptoms, sickness absences, and reduced job performance (Clark, Michel, Zhdanova, Pui, \& Baltes, 2016; Falco et al., 2013). More specifically, given that individuals with strong 
narcissistic components pursue power, status, social recognition, and rewards in the workplace, they may dedicate an excessive amount of time and effort to their work, at the expense of other life activities. Accordingly, we hypothesized narcissism to be positively associated with workaholism. Furthermore, building on the biopsychosocial model (McMillan \& O'Driscoll, 2008), we also hypothesized that workload, an aspect of the job that pushes employees to work hard (i.e., a situational factor), may moderate the association between narcissism (i.e., an individual factor) and workaholism, which is expected to be stronger when workload is high.

\subsection{Workaholism and the biopsychosocial model}

Workaholism may be defined as "the tendency to work excessively hard in a compulsive way" (Schaufeli, Taris, \& Bakker, 2008, p. 10). Working excessively (WE) and working compulsively (WC) are the two core dimensions of the construct, and workaholism is characterized by high levels of both. According to the biopsychosocial model (McMillan \& O'Driscoll, 2008), workaholism has a multifactorial genesis. From this standpoint, it may stem from a complex interaction between personal dispositions (e.g., personality traits), cognitive and emotional processes (e.g., rigid beliefs, such as performance-based self-esteem), behaviors learned by the individuals (e.g., in the family, at work), and the social systems (e.g., the work context) in which they are embedded (see Andreassen, 2014, for a review). Hence, in line with the biopsychosocial model, personality traits such as narcissism may predispose individuals to workaholism, which is triggered by work-related factors (e.g., workload) and then maintained by cognitive and behavioral dysfunctional patterns.

\subsection{Narcissism, workaholism, and the moderating role of workload}

Among the individual factors that may give rise to workaholism, narcissism could play a central role. Narcissism can be conceived "as an important complex of personality traits and processes that involve a grandiose yet fragile sense of self and entitlement as well as a preoccupation with success and demands for admiration" (Ames, Rose, \& Anderson, 2006, p.440). A possible mechanism that explains the association between narcissism and workaholism could involve work motivation. For example, drawing on the Self-Determination Theory (SDT; Ryan \& Deci, 2000), van Beek, Taris, and Schaufeli (2011) found that workaholic employees were mostly driven by controlled motivation, in terms of external and introjected regulation. This implies that workaholics mainly perform their work for its instrumental value. More specifically, workaholics work extremely hard to attain social rewards or to avoid punishment, such as social recognition or disapproval by others in the workplace, respectively (i.e., external regulation). They also work hard to meet internalized external standards of self-worth and social approval, in order to enhance feelings of worth and self-esteem as well as to avoid negative emotions related with failure (i.e., introjected regulation).

Interestingly, previous research suggested narcissism to be positively associated with controlled motivation (Sedikides, Ntoumanis, \& Sheldon, 2019). Individuals with strong narcissistic components are characterized by a chronic need to attain validation for their overly positive yet fragile self-image (Morf, Weir, \& Davidov, 2000). Hence, narcissists may work extremely hard to acquire external rewards and appreciation from others (e.g., social recognition, from colleagues or supervisor), as well as to avoid disapproval (i.e., external regulation). Moreover, individuals with strong narcissistic components may find themselves in a persistent state of ego-involvement, in which their self-esteem is contingent on their performance (Morf et al., 2000). These individuals may, therefore, feel pushed to work exceedingly hard, to enhance feelings of self-worth and to avoid negative emotions related with failure, such as anxiety and guilt (i.e., introjected regulation). Overall, based on the arguments previously described and the results of previous research (Clark et al., 2010), we hypothesized that narcissism is positively associated with workaholism as well as its dimensions of WE and WC.

Hypothesis 1a: Narcissism is positively associated with workaholism;

Hypothesis 1b: Narcissism is positively associated with WE;

Hypothesis 1c: Narcissism is positively associated with WC.

Based on the biopsychosocial model, we expect workload to moderate the above-mentioned association between narcissism and workaholism. Indeed, workload may signal what the norms are in an organization or in a work team, that is, to work extremely hard (Andreassen et al., 2017). Hence, high workload may encourage workaholism in employees with strong narcissistic components, who may feel pushed to work hard to achieve social recognition and rewards, as well as to attain ego enhancement, by meeting these extremely demanding external standards. Overall, based on these arguments and given the assumptions of the biopsychosocial model, we hypothesized that workload moderates the association between narcissism and workaholism as well as its dimensions (i.e., WE and WC), with this association being stronger for workers with higher workload. 
Hypothesis 2a: Workload moderates the association between narcissism and workaholism; Hypothesis 2b: Workload moderates the association between narcissism and WE; Hypothesis 2c: Workload moderates the association between narcissism and WC.

\section{Methods}

The study was conducted among workers from different organizations in Italy $(N=217)$. The sample consisted of 121 women and 95 men (one missing value), with a mean age of 45 years $(S D=12)$. Participants completed a self-report questionnaire (paper and pencil) aimed at determining narcissism, workaholism, and workload. The questionnaire was administered anonymously, and participants took part in the study voluntarily.

\subsection{Measures}

The following self-report measures were administered.

Narcissism was assessed using an Italian adaptation (13 items) of the short form of the Narcissistic Personality Inventory (NPI-16; Ames et al., 2006). Narcissism-consistent responses were coded as 1 whereas narcissism-inconsistent responses were coded as 0 , and then an ovall narcissism score was computed.

Workaholism was assessed using the Italian adaptation (Kravina, Falco, Girardi, \& De Carlo, 2010) of the Dutch Work Addiction Scale (DUWAS; Schaufeli et al., 2008). The scale includes ten items aimed at determining the two dimensions of working excessively (six items) and working compulsively (four items). The response scale ranged from 1 (strongly disagree) to 6 (strongly agree).

Workload was assessed using three items taken from the $\mathrm{Q}_{\mathrm{u}}$-Bo test, an instrument standardized for the Italian context (De Carlo, Falco, \& Capozza, 2008). The response scale ranged from 1 (strongly disagree) to 6 (strongly agree).

\subsection{Data analysis}

The hypothesized relationships were tested using moderated multiple regressions analyses (Aiken \& West, 1991). Overall, three different models were estimated. In Model 1 (M1), workaholism was the dependent variable, whereas narcissism and workload were the independent and the moderating variables, respectively. Model 2 (M2) and Model 3 (M3) were similar to M1, except that working excessively (M2) or working compulsively (M3) where the dependent variables. If a significant interaction was found, then a simple slope analysis was conducted, to determine whether narcissism is associated with workaholism at high $(+1 S D)$ and low $(-1 S D)$ levels of workload. The analyses were conducted using the software R (R Core Team, 2019).

\section{Results}

Results showed that narcissism was not associated with either workaholism (overall score, M1) or its dimensions, namely WE and WC (M2 and M3, respectively). Our first hypothesis was not supported. Furthermore, workload moderated the association between narcissism and the overall workaholism score in M1 $(b=.07, p<.001)$. Similarly, workload moderated the association between narcissism and WE $(b=.05, p<.05)$ as well as the association between narcissism and WC $(b=.08$, $p<.001)$. The interaction between narcissism and workload accounted for an additional $4.3 \%$ of the variance in workaholism (M1), 2.1\% in WE (M2), and 5.3\% in WC (M3). Simple slope analysis revealed that the relationship between narcissism and workaholism (overall score, M1) was positive and significant only when workload was high $(b=.12, p<.001)$. In a similar way, the relationship between narcissism and WE $(b=.11, p<.01)$ as well as that between narcissism and $\mathrm{WC}(b=.14, p<.001)$ were positive and significant only when workload was high. Overall, our second hypothesis was supported.

\section{Discussion}

This study investigated the association between narcissism and workaholism. Building on the biopsychosocial model (McMillan \& O'Driscoll, 2008), it was hypothesized that narcissism is positively associated with workaholism, with workload moderating this relationship, which is expected to be stronger when workload is high. Results partially supported our predictions. In fact, narcissism was positively associated with workaholism only in individuals facing high workload. Accordingly, our results suggest that narcissism per se may not give rise to workaholism. However, narcissism may be a risk factor for workaholism in the presence of a demanding work environment. 
A possible explanation for this pattern of association is that in a work environment characterized by low or moderate levels of stress (e.g., when workload is low or moderate) individuals with strong narcissistic components may inherently approach and enjoy their work, in which they can satisfy their need of power and admiration as well as their strong desire of demonstrating superiority over others (e.g., colleagues; Clark et al., 2010). In other words, in these situations, narcissism may be associated with work engagement (a "good" form of heavy work investment), not workaholism (a "bad" form of heavy work investment; Schaufeli, 2016). This is consistent with a previous study by Andreassen et al. (2012), who found narcissism to be positively associated with enjoyment of work and work engagement, but unrelated to drive, a core characteristic of workaholism that is especially associated with negative outcomes for the individual (e.g., emotional exhaustion; Van den Broeck et al., 2011).

However, in a stressful work environment (e.g., when workload is high) narcissism may lead to the onset of workaholism. In these situations, individuals with strong narcissistic components, who are characterized by a chronic need to attain validation for their overly positive yet fragile self-image (Morf et al., 2000), may feel pushed to work exceedingly hard to achieve social recognition and rewards, as well as to attain ego enhancement, by meeting the extremely demanding external standards (e.g., elevated expectations in terms of productivity and performance). In other words, when workload is high narcissism may play a role in the development of an obsessive inner drive to work hard. Interestingly, previous research on the relationship between perfectionism and workaholism has shown similar results. For example, Falco et al. (in press) found in a longitudinal study that self-oriented perfectionism predicted an increase in workaholism over time only in workers facing high workload. Taken together, these results are in line with the predictions of the biopsychosocial model (McMillan \& O'Driscoll, 2008), according to which workaholism can be conceived as the product of a complex interaction between individual (e.g., personality traits) and situational (e.g., organizational) factors.

Finally, from a practical standpoint, this study suggests that organizations should target narcissism, especially in managers, who necessarily face high job demands in their job. Narcissistic leaders are particularly at risk of workaholism and may also encourage workaholism in their subordinates. When dealing with narcissism in organizational contexts, preventive measures are suggested to be much more effective than curative ones (Fuller, Galvin, \& Ashforth, 2018). In general terms, interventions aiming at reducing the negative consequences of individuals' narcissistic traits and characteristics should foster the development of an organization's culture that stigmatizes self-serving and narcissistic behaviors and promotes calm collaboration among managers and co-workers (Fuller et al., 2018).

\section{Conclusion}

Overall, this study suggests that individuals with strong narcissistic components can be at risk of workaholism when workload is high. From a theoretical perspective, this is consistent with the idea that workaholism may stem from a complex interaction between individual and situational factors. From a practical standpoint, interventions aimed at preventing workaholism should target narcissism in workers who face high levels of workload, such as managers.

\section{References}

Aiken, L. S., \& West, S. G. (1991). Multiple regression: Testing and interpreting interactions. Thousand Oaks, CA, US: Sage Publications, Inc.

Ames, D. R., Rose, P., \& Anderson, C. P. (2006). The NPI-16 as a short measure of narcissism. Journal of Research in Personality, 40, 440-450. doi:10.1016/j.jrp.2005.03.002

Andreassen, C. S. (2014). Workaholism: An overview and current status of the research. Journal of Behavioral Addictions, 3, 1-11. doi:10.1556/JBA.2.2013.017

Andreassen, C. S., Bakker, A. B., Bjorvatn, B., Moen, B. E., Magerøy, N., Shimazu, A., ... Pallesen, S. (2017). Working conditions and individual differences are weakly associated with workaholism: A 2-3-year prospective study of shift-working nurses. Frontiers in Psychology, 8. doi:10.3389/fpsyg.2017.02045

Andreassen, C. S., Ursin, H., Eriksen, H. R., \& Pallesen, S. (2012). The relationship of narcissism with workaholism, work engagement, and professional position. Social Behavior and Personality: An International Journal, 40, 881-890. doi:10.2224/sbp.2012.40.6.881

Campbell, W. K., Hoffman, B. J., Campbell, S. M., \& Marchisio, G. (2011). Narcissism in organizational contexts. Human Resource Management Review, 21, 268-284. doi:10.1016/j.hrmr.2010.10.007 
Clark, M. A., Lelchook, A. M., \& Taylor, M. L. (2010). Beyond the Big Five: How narcissism, perfectionism, and dispositional affect relate to workaholism. Personality and Individual Differences, 48, 786-791. doi:10.1016/j.paid.2010.01.013

Clark, M. A., Michel, J. S., Zhdanova, L., Pui, S. Y., \& Baltes, B. B. (2016). All work and no play? A meta-analytic examination of the correlates and outcomes of workaholism. Journal of Management, 42, 1836-1873. doi:10.1177/0149206314522301

De Carlo, N. A., Falco, A., \& Capozza, D. (2008). Test di valutazione del rischio stress lavoro-correlato nella prospettiva del benessere organizzativo, $Q_{u}$-Bo [Test for the assessment of work-related stress risk in the organizational well-being perspective, $\mathrm{Q}_{\mathrm{u}}-\mathrm{Bo}$ ]. Milano, Italy: FrancoAngeli.

Falco, A., Girardi, D., Dal Corso, L., De Carlo, A., \& Di Sipio, A. (in press). Does workload moderate the association between perfectionism and workaholism? A longitudinal study. Journal of Personnel Psychology.

Falco, A., Girardi, D., Kravina, L., Trifiletti, E., Bartolucci, G. B., Capozza, D., \& De Carlo, N. A. (2013). The mediating role of psychophysic strain in the relationship between workaholism, job performance, and sickness absence: A longitudinal study. Journal of Occupational and Environmental Medicine, 55, 1255-1261. doi:10.1097/JOM.0000000000000007

Fuller, P. J., Galvin, B. M., \& Ashforth, B. E. (2018). Larger than life: Narcissistic organizational identification in leadership. Organizational Dynamics, 47, 8-16. doi:10.1016/j.orgdyn.2017.06.003

Kravina, L., Falco, A., Girardi, D., \& De Carlo, N. A. (2010). Workaholism among management and workers in an Italian cooperative enterprise. TPM - Testing, Psychometrics, Methodology in Applied Psychology, 17, 201-216. doi:10.4473/TPM.17.4.2

McMillan, L. H. W., \& O'Driscoll, M. P. (2008). The wellsprings of workaholism: A comparative analysis of the explanatory theories. In R. J. Burke \& C. L. Cooper (Eds.), The long work hours culture. Causes, consequences and choices (pp. 85-111). Bingley, UK: Emerald Group Publishing Limited.

Morf, C. C., Weir, C., \& Davidov, M. (2000). Narcissism and intrinsic motivation: The role of goal congruence. Journal of Experimental Social Psychology, 36, 424-438. doi:10.1006/jesp.1999.1421

R Core Team. (2019). R: A Language and environment for statistical computing. Vienna, Austria: $\mathrm{R}$ Foundation for Statistical Computing.

Ryan, R. M., \& Deci, E. L. (2000). Intrinsic and extrinsic motivations: Classic definitions and new directions. Contemporary Educational Psychology, 25(1), 54-67. doi:10.1006/ceps.1999.1020

Schaufeli, W. B. (2016). Heavy work investment, personality and organizational climate. Journal of Managerial Psychology, 31, 1057-1073. doi:10.1108/JMP-07-2015-0259

Schaufeli, W. B., Taris, T. W., \& Bakker, A. B. (2008). It takes two to tango: Workaholism is working excessively and working compulsively. In R. J. Burke \& C. L. Cooper (Eds.), The long work hours culture. Causes, consequences and choices (pp. 203-225). doi:10.1016/B978-1-84855-0384.00009-9

Sedikides, C., Ntoumanis, N., \& Sheldon, K. M. (2019). I am the chosen one: Narcissism in the backdrop of self-determination theory. Journal of Personality, 87, 70-81. doi:10.1111/jopy.12402

Snir, R., \& Harpaz, I. (2012). Beyond workaholism: Towards a general model of heavy work investment. Human Resource Management Review, 22, 232-243. doi:10.1016/j.hrmr.2011.11.011

van Beek, I., Taris, T. W., \& Schaufeli, W. B. (2011). Workaholic and work engaged employees: Dead ringers or worlds apart? Journal of Occupational Health Psychology, 16, 468-482. doi: $10.1037 / \mathrm{a} 0024392$

Van den Broeck, A., Schreurs, B., De Witte, H., Vansteenkiste, M., Germeys, F., \& Schaufeli, W. B. (2011). Understanding workaholics' motivations: A self-determination perspective. Applied Psychology, 60, 600-621. doi:10.1111/j.1464-0597.2011.00449.x 